\section{Are centromeric dots kinetochores?}

Elberg ${ }^{1}$ described a Giemsa staining technique which reveals specific paired dots in the centromere region of human chromosomes in cells treated with colcemid and hypotonic $\mathrm{KCl}$. He suggested that the dots may represent organelles associated with spindle fibres. Evans and Ross $^{2}$ demonstrated similar, stained or unstained dots obtained in colcemidtreated mammalian cells by other preparatory techniques. They hypothesised that these dots "may represent the kinetochores and particularly their associated proteins"

These interpretations are unlikely for several reasons. First, the location of the unstained dots shown by Evans and Ross ${ }^{2}$ does not coincide with that of the raised dots on unstained, shadowed chromosomes. Unstained dots adjoin chromosomes in the centromere region, whereas raised dots, like Eiberg's ${ }^{1} C_{d}$ dots, lie clearly within the chromosomes. Second, there is no evidence that the centromerically located spindle microtubules are always present in colcemid-treated cells. A two hour exposure of rat kangaroo cells to $0.05 \mathrm{Hg} \mathrm{ml}^{-1}$ colcemid destroys all microtubules and alters the fine structure of the kinetochores ${ }^{3}$. Speculation that spindle proteins concentrated in the centromere regions of chromosomes could produce centromeric dots cannot, therefore, be substantiated. Third, kinetochores are rather delicate, labile organelles and they are probably affected drastically by treatments used in the preparation of chromosomes for light microscopy. For example, no kinetochores are visible in thin sections of Chinese hamster chromosomes subjected to the aceto-orcein smear technique (L. J. Journey, personal communication). Fourth, even if kinetochores were preserved by light microscopic techniques they could hardly produce prominent raised dots, for the disk-shaped kinetochores of mammalian chromosomes are only some $100 \mathrm{~nm}$ thick ${ }^{3.4}$.

Optical and electron microscopic observations of rat kangaroo cells treated with hypotonic colcemid ${ }^{5}$ suggest a different interpretation of centromeric dots. Paired dots are recognisable by phase contrast microscopy in the centromere region of favourably oriented chromosomes in embedded prometaphase cells (Fig. Ia). In thin sections the dots appear as patches of chromatin packed more densely than in the remainder of the chromosomes (Fig. 1b). The kinetochores which are less opaque to electrons lie adjacent to these patches. In metaphase cells the chromosomes are, overall, more condensed, but the chromatin is also more densely packed in the centromere region than in the arms (Fig. 1c). The outer layer of each kine-

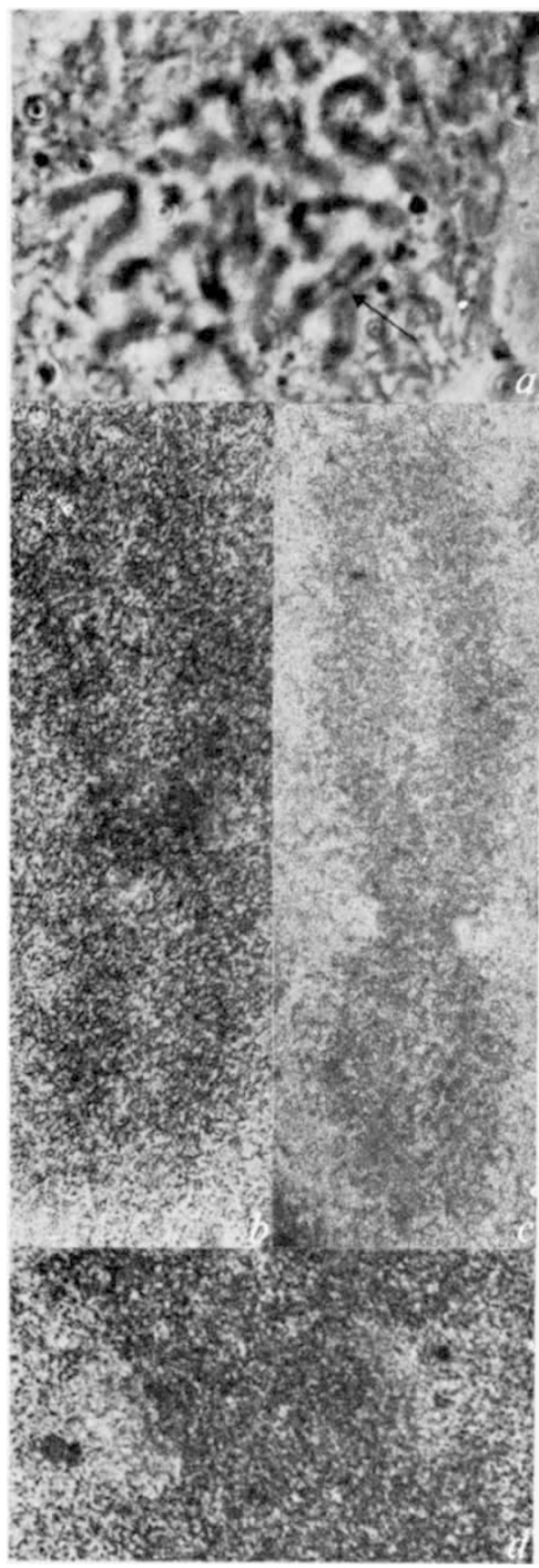

tochore appears as a moderately electronopaque band lying parallel to the chromosomal surface in a distinctly electronlucent, approximately circular zone adjacent to the centromere region (Fig. $1 c$ and $d$ ).

These electron-lucent zones correlate with, and may be equivalent to, the unstained dots described by Evans and Ross ${ }^{2}$. This interpretation is supported by Journey's observations (personal communication) of "empty", circular zones adjacent to the centromere region of Chinese hamster chromosomes in thin sections of acetoorcein smears. The densely packed centromeric chromatin, on the other hand, may account for the dots revealed by Eiberg's ${ }^{1}$ Giemsa staining technique and by the shadowing technique of Evans and Ross ${ }^{2}$. Dense structures reminiscent of centromeric dots are also seen in the centromere region in wholemount preparations of Chinese hamster chromosomes treated with distilled water ${ }^{6}$ and in whole mounts of HeLa chromosomes stained with phosphotungstic acid? All these results can be explained by a greater resistance of the centromeric chromatin to the spreading forces to which chromosomes are subjected in a hypotonic medium. This resistance may reflect a specific DNA-protein composition of the centromeric chromatin, that in turn could account for the specific staining, as pointed out by Eiberg ${ }^{1}$.

\section{U.-P. Roos}

Département de Biologie Végétale

University of Geneva,

1211 Geneva 4, Switzerland

1 Eiberg, H., Nature, 248, 55 (1974).

2 Evans, H. J., and Ross, A., Nature, 249, 861-862 (1974).

Roos, U.-P., Chromosona, 41, 195-220 (1973).

4 Jokelainen, P. T., J. Ultrastruct. Res., 19, 19-44 (1967).

Roos, U.-P., thesis, Univ. Florida (1971).

- Stubblefield, E., and Wayne, W., Chromosoma, 32, 262-294 (1971).

Moses, M. J. and Counce, S. J., J. exp. Zool., 189, $115-120(1974)$.

Fig. 1 Chromosomes of rat kangaroo cells (line $\mathrm{PtK}_{2}$ ) treated with hypotonic colcemid (15 min at $37^{\circ} \mathrm{C}$ in $0.25 \mu \mathrm{g}$ $\mathrm{nl}^{-1}$ colcemid prepared by diluting an aqucous $0.5 \mu \mathrm{g} \mathrm{mi}^{-1}$ stock solution $\mathrm{l}: 1$ with culturc medium. See ref. 3 for culture conditions, fixation and cmbedding). a. Phase contrast micrograph of a prometaphasecell $(\times 1,625)$. Note the paired dots in the centromere region of favourably oriented chromosomes. Single dots are visible on laterally viewed chromosomes. $b$, Thin section of the arrowed chromosome of Fig. la $\times 11,700$. Note the two patches of densely packed chromatin in the centromere region. $c$ Metaphase chromosome $\times 7,475$. Note the dense centromeric chromatin and the adjoining electron-lucent zones. d, Centromere region of another metaphase chromosome $\times 22,425$. Note the kinetochore bands at the surface of the primary constriction and the electronlucent zones.

\section{Logic of animal conflict} that it is not necessary to invoke group selection to explain the occurrence of "conventional' rather than 'dangerous' tactics in animal conflict. The conditions for the evolution, under individual selection, of populations in which conventional tactics predominate are, however, more stringent than they suggest. In particular, given their pay-off matrix, the population may end up consisting mainly of individuals adopting the 'dangerous' strategies, Hawk and Bully.

Consider a population consisting almost entirely of Hawk and Bully, so that nearly all conflicts are between these. Then
MAYNARD-SMITH and Price have shown ${ }^{1}$ 